Cahiers $d u$ MONDE RUSSE

\section{Cahiers du monde russe}

Russie - Empire russe - Union soviétique et États indépendants

$49 / 4 \mid 2008$

Destins individuels et terreur. Jeunesse dans la société post-stalinienne

\title{
Dynamiques et contraintes de la critique à l'époque stalinienne
}

Traces des pratiques communicatives dans le journal d'A.G. Man'kov

\section{Malte Griesse}

\section{(2) OpenEdition}

\section{Journals}

Édition électronique

URL : https://journals.openedition.org/monderusse/9497

DOI : 10.4000/monderusse. 9497

ISSN : $1777-5388$

Éditeur

Éditions de l'EHESS

Édition imprimée

Date de publication : 28 décembre 2008

Pagination : 605-628

ISBN : 978-2-7132-2197-2

ISSN : $1252-6576$

\section{Référence électronique}

Malte Griesse, "Dynamiques et contraintes de la critique à l'époque stalinienne », Cahiers du monde russe [En ligne], 49/4 | 2008, mis en ligne le 01 janvier 2008, consulté le 02 septembre 2022. URL http://journals.openedition.org/monderusse/9497 ; DOI : https://doi.org/10.4000/monderusse.9497 


\title{
DYNAMIQUES ET CONTRAINTES DE LA CRITIQUE À L'ÉPOQUE STALINIENNE
}

\author{
Traces des pratiques communicatives \\ dans le journal d'A.G. Man'kov
}

7.4.1933. La crise économique est réelle [... ce qui] contraint notre gouvernement à prendre une série de mesures radicales, qu'il copie servilement dans les modèles du passé. [...] La baisse des salaires est appliquée sans la moindre explication. [...] Tout cela est cyniquement présenté dans les journaux comme une nouvelle orientation vers le «meilleur et honnête travailleur et udarnik», vers « l'actif du parti progressiste (peredovoj) ».

Qu'en est-il en réalité ? ! L'État est le premier spéculateur et le premier débiteur. [...] Il n'y a personne dans toute l'Union soviétique à qui l'État ne doive pas quelque chose. [...]

Si l'on sait que les produits rationnés délivrés pour un mois suffisent à peine pour couvrir les besoins de trois à quatre jours, [...] on peut facilement imaginer la faiblesse du niveau de vie de la majorité de la population [...].

Comment cet arbitraire est-il possible ? Par le renforcement de la dictature militaire ! Ce n'est pas un secret que les deux tiers du budget de l'État sont gaspillés par l'armée, pour la soldatesque [...].

[...] dans les provinces du sud (plus riches en blé !) les gens ont le ventre enflé par la faim et cherchent à gagner les villes. Dans ces mêmes villes on lance entre 80 et 120 roubles à la majorité de la population, tout en la condamnant à la misère et à la faim, alors que dans l'armée on introduit des cartes de rationnement privilégiées, en d'autres termes, une forme de salaire en nature. ${ }^{1}$

1. Extrait du Journal d'A.G. Man'kov. Dans les références nous nous appuyons sur la version publiée par l'auteur dans les années 1990 : A.G. Man’kov, « Iz dnevnika rjadovogo čeloveka. (1933-1934gg.) » [A.G. Man'kov, Extraits du journal d'un homme ordinaire, (1933-1934)], Zvezda, 5 (1994), p. 134-183 et «Iz Dnevnika 1938-1941gg. » [Extraits du journal (19381941], Zvezda, 11 (1995), p. 167-199. Les traductions des passages présentés dans cet article sont les nôtres. Plus tard Man'kov a de nouveau publié les mêmes extraits de son journal sous forme d'un petit livre, Dnevniki tridcatyh godov [Journaux des années 1930], SPb., 2001(serija 
Dans le journal du futur historien et spécialiste des $\mathrm{XVI}^{\mathrm{e}}-\mathrm{XVII}{ }^{\mathrm{e}}$ siècles, Arkadij Man'kov (1913-2006), on trouve maints passages de ce genre qui dénoncent sans ambages la propagande officielle stalinienne et rompent avec ses grands tabous.

Le diariste condamne l'exploitation de la population (surtout des ouvriers) par l'État (sa prétendue « dictature du prolétariat ») et, par voie de conséquence, dénonce le système stalinien comme variante du capitalisme, d'un capitalisme « dans sa pire phase, celle de l'appauvrissement et de la décadence » qui ressemblerait tout à fait à «l'accumulation initiale » du capital que des dizaines de millions de gens auraient payée de leur sang. Pour les dirigeants du pays, soi-disant « socialistes », le nom de Marx ne serait qu'une feuille de vigne, tandis qu'en réalité leur politique consisterait à «passer par-dessus les peuples et les générations ». La subvention de l'industrie lourde au détriment des biens de consommation, impliquant une croissance déséquilibrée entre les différents secteurs économiques, n'aurait rien à voir avec le socialisme, et la rhétorique officielle ne renverrait qu'à un « pseudo-marxisme » [30.3.1933].

Sans doute, il s'agit là d'un des documents personnels les plus critiques qui soient conservés des années 1930. Envergure et portée de la critique sont d'autant plus remarquables que le diariste de vingt ans a grandi sous les bolcheviks et ne dispose donc pas d'une échelle de valeurs relevant d'un autre ordre. Ses parents sont loin d'être des adhérents enthousiastes de Stalin. Son père, secrétaire en chef du département civil du Sénat sous l'ancien régime², a assisté à l'avènement de la révolution avec réticence. Mais ses parents ne sont pas des opposants. Dans son journal, il leur reproche fréquemment leur lâcheté, et de ne pratiquement jamais parler de politique, même pas à la maison.

Selon les souvenirs du diariste - qui a lui-même édité les deux seuls cahiers conservés (1933-1934 et 1938-1941) —, son journal des années 1920 était marqué par l'enthousiasme pour la révolution, pour Lenin, le $1^{\mathrm{er}}$ Mai, etc. L'orientation critique générale du journal des années 1930 ne contredit pas cette tendance: comme pratiquement tous les critiques du régime stalinien, Man'kov s'appuie sur les valeurs de la révolution pour les opposer à la réalité, au «cynisme » et aux «mensonges » de la propagande des années $1930^{3}$. Aussi ses opinions ne sont-elles pas le reflet des attitudes de ses taciturnes parents.

«Dnevniki i vospominanija peterburgskih učenyh»). En tant qu'historien, Man'kov travaillera entre autres sur les révoltes sociales (notamment le Temps des Troubles, mais aussi sur le soulèvement de Razin), sur la législation (son édition de l'Uloženie de 1649 est particulièrement bien connue), sur l'économie des biens patrimoniaux et les prix au XVII siècle.

2. «Ober-sekretar' graždanskogo departamenta Senata ». Voir l'entrée du 13.4.1933 sur la vie de son père, un officier tsariste. Yasuhiro Matsui, « Youth Attitudes towards Stalin's Revolution and the Stalinist Regime, 1929-41 », Acta Slavica Iaponica, Tomus XVIII (2001), p. 6478, p. 75.

3. Ceci vaut, bien entendu, pour les trotskistes et les autres opposants déclarés, mais aussi pour les ouvriers en grève et le fondement de leurs revendications vis-à-vis du régime Jeffrey J. Rossman, Worker Resistance under Stalin: Class and Revolution on the Shop Floor, Cambridge, MA-Londres : Harvard University Press, 2005 et, même à l'extérieur, pour beaucoup de renégats et de réfugiés (Michael Voslensky, Nomenklatura: The Soviet Ruling Class, Garden City, NY : Doubleday, 1984). Même si l'on observe des manifestations critiques à une échelle plus intime, cette impression est généralement confirmée. Par critique nous n'entendons 
De la première lecture se dégage l'indépendance du jugement de Man'kov qui développe sa critique du régime à partir de ses propres ressources, sa propre observation, sa propre réflexion. Man'kov pourrait ainsi être considéré comme un individu autonome résistant aux pièges et aux tentations de la propagande totalitaire et c'est ainsi qu'il est présenté par la rédaction de Zvezda qui publie son journal dans les années 1990. C'est aussi, dans le même numéro de cette revue, la teneur du courrier d'un lecteur qui célèbre ces individus qui, comme Man'kov, n'ont pas succombé à la toute-puissance du système, même s'ils ne pouvaient pas exprimer publiquement leurs critiques. Le journal de Man'kov témoignerait des considérations tacites de beaucoup d'autres individus solitaires à cette époque où toute hétérodoxie fut sévèrement réprimée. Il s'agit de la figure de l'émigration intérieure, bien connue en Allemagne depuis la fin du régime nazi ${ }^{4}$.

Et ce qui est conservé du journal de Man'kov commence en effet par une sorte de bilan de ses dix-huit mois passés à l'usine Triangle rouge. "C'est précisément là que s'est formée ma conception du monde (mirosozercanie), que j'ai compris la situation du pays par le prisme de la vie à l'usine » [30.3.1933]. Malgré son aversion pour un travail d'employé qu'il juge complètement inutile, l'usine fut pour lui une «école de vie », un microcosme qui lui a appris à mieux saisir le sens de l'ensemble. Par sa « conception du monde », Man'kov entend son attitude critique à l'égard du régime et du système stalinien. Il s'imagine ainsi comme un observateur extérieur ayant étudié le cœur du système (une usine, prise comme pars pro toto), pour en tirer ses conclusions et jugements. Selon lui, ce serait précisément son détachement qui l'aurait aidé à parvenir à des évaluations indépendantes et impartiales.

Une seconde lecture, quasiment opposée à la première, est proposée par le courant des études sur la subjectivité qui s'interroge sur les façons dont les sujets s'inscrivent activement dans l'idéologie communiste et notamment dans le projet de la création d'un Homme nouveau, projet collectif particulièrement propice à l'adoption en modèle individuel. Par le biais de la refonte de soi, les sujets s'engageraient de plus en plus dans la logique du pouvoir, de sorte que leur identité personnelle dépend de plus en plus de l'acteur historique collectif dont le régime est le seul garant. En conséquence, tout mouvement critique contre le régime au pouvoir se tourne inéluctablement contre les critiques dans la mesure où il sape le soubassement même de leur nouvelle identité. Chez Man'kov cette dépendance s'exprimerait dans ses accès de doute de soi-même. Par ailleurs, en appuyant sa

pas tous les grognements inarticulés (comme dans les files d'attente) à propos de tel ou tel mal, mais des jugements qui transcendent le particulier pour envisager le général. Une critique marchande-libérale n'apparaît pratiquement jamais. Sans que cela détermine forcément les comportements des gens, c'est d'une importance énorme pour la construction d'un ordre légitime. Voir Luc Boltanski, Laurent Thévenot, De la justification : Les économies de la grandeur, P. : Gallimard 1991. Plus généralement, les réflexions sur la jonction micro-macro dans l'argumentation des acteurs, en l'occurrence de Man'kov, doivent beaucoup à nos entretiens avec Laurent Thévenot, ainsi qu'à son livre plus récent, L'action au pluriel : Sociologie des régimes d'engagement, P. : Éditions la Découverte 2006.

4. Zvezda, 11, 1995, p. 167. 
critique sur la révolution et des schémas interprétatifs marxistes, il ferait appel aux mêmes catégories que le système stalinien et ne ferait en dernière instance qu'en renforcer la vigueur. L'argument vient d'une théorie critique du pouvoir, telle que l'a proposé Michel Foucault, notamment à l'égard des sociétés occidentales 5 .

À la différence de ces deux approches dont l'une met l'accent sur l'autonomie du jugement de l'individu critiquant, et la seconde fait dépendre la subjectivité du discours officiel, dans le présent article nous allons montrer dans un premier temps que les jugements critiques de Man'kov s'appuient essentiellement sur des engagements mutuels avec des personnes de son entourage qui lui permettent de communiquer et d'élaborer ses opinions (en l'occurrence surtout politiques). Sous l'emprise du système stalinien et de ses nombreuses instances de contrôle, cette communication ne procède que rarement d'un échange sur un espace public critique. Elle repose sur une variété de niveaux de communication, depuis l'intimité jusqu'à des cercles étendus à un milieu professionnel, en passant par des liens amicaux. Pour cela nous porterons attention à l'architecture de ces engagements entre eux, du plus intime au plus public, qui correspondent à des possibilités diverses de communiquer un mécontentement, depuis le mouvement d'humeur jusqu'à la critique argumentée appropriée à un jugement universalisable.

Dans un deuxième temps, nous juxtaposerons les deux extraits conservés du journal qui correspondent à deux phases cruciales et bien distinctes du développement du système soviétique sous Stalin : la famine avant l'assassinat de Kirov et l'avant-guerre après l'apogée de la Terreur. Nous mettrons ainsi en évidence un changement important dans la communication relative à l'entourage du diariste. Celle-ci nous semble refléter le changement d'atmosphère plus général dans la société soviétique, surtout après la signature du pacte germano-soviétiqué6.

5. Dans son étude riche et stimulante, Jochen Hellbeck (Revolution on my Mind: Writing a Diary under Stalin, Cambridge, MA : Harvard University Press, 2006) interprète des journaux intimes des années 1930 sous cet angle et aboutit en quelque sorte à un renouveau du modèle totalitaire, mais avec la prise en compte du rôle actif d'un « sujet » historiquement malléable. Si le propos est d'historiciser le sujet, la notion du système et du « discours » semble assez monolithique. Le modèle foucaldien d'un pouvoir discursif diffus omniprésent et passant par la subjectivité explique mal le déploiement de la Terreur. Nous nous proposons donc d'examiner de plus près la dynamique inhérente aux différences des prises de position et des argumentations qui rendent le régime beaucoup moins sûr de lui-même que ne le suggère l'idée d'un État totalitaire. À notre avis, l'équilibre du pouvoir, si toutefois il en existait un en Union soviétique sous Stalin, reste toujours fragile et c'est de là qu'il faut partir pour retracer la genèse de la Terreur.

6. Il ne sera pas question dans cet article de présenter Man’kov comme représentant d'une catégorie sociale, professionnelle ou culturelle donnée pour explorer les mentalités de ce groupe, tel que le fait l'histoire sociale. Les catégories dont il relève, comme celles de la jeunesse, des employés, des ouvriers, de la profession d'historien, etc. ne sont observées qu'au moment où le diariste lui-même cherche à les faire valoir dans son argumentation. Pour des études portant sur des catégories plus substantialistes voir, par exemple, pour la jeunesse, Corinna Kuhr-Korolev, Monica Wellmann, Stefan Plaggenborg, éds., Sowjetjugend 19171941: Generation zwischen Revolution und Resignation, Essen, 2001; pour les ouvriers Donald A. Filtzer, Soviet Workers and Stalinist Industrialization : The formation of modern Soviet production relations, 1928-1941, London: Pluto Press, 1986 ; pour la profession d'historien, A.M. Dubrovskij, Istorik $\mathrm{i}$ vlast' : Istoričeskaja nauka v SSSR i koncepcija istorii 


\section{Le dialogue comme catalyseur de la critique}

L'effort de synthèse du début de ce qui nous reste du journal débouche sur une dénonciation du système soviétique des années 1930 [30.3.1933]. Il est né d'une dispute avec Aleša Gorškov, un ami depuis leur enfance à Kašin [18.2.1934]. Ils ne se sont pas vus depuis deux mois lorsque Gorškov se présente au cours supérieur de bibliothéconomie que suit Mańkov à la bibliothèque publique, conjointement à son travail au Triangle rouge. Gorškov est pâle et a les joues creuses :

Nous nous sommes à tel point plongés dans la conversation que finalement nous avons parlé pendant tout le cours de bibliographie. Il [Gorškov] a été licencié de son travail. Qui plus est, on ne lui a pas accordé de passeport et il sera donc obligé de quitter Leningrad. Il dit : "Les salauds, ils me gâchent la vie. Ils me renvoient là d'où je viens et il faudra me frayer un nouveau chemin. » L'année prochaine il devra partir à l'armée. Néanmoins, notre dispute a porté sur l'actualité. [30.3.1933]

La suite, un développement élaboré, point par point, de critique du régime, laisserait croire que tout était déjà prêt dans son esprit, que les arguments et jugements étaient le résultat d'une réflexion solitaire préalable dont il suffisait de faire part à un interlocuteur. Mais le passage est introduit par un commentaire révélateur: « Nerveux, sentant que je parlais de manière embrouillée et contradictoire, je lui ai démontré... » : la systématisation et le résumé des arguments que l'on trouve dans le journal ne sont que le résultat de l'échange d'idées, d'expériences, de perceptions et d'opinions avec l'ami. La critique s'est forgée, ou du moins consolidée et nuancée, au cours de cette tentative de convaincre Gorškov, même si (ou peut-être parce que) cette tentative s'est (d'abord) révélée vaine :

Gorškov reconnaît les faits, mais il n'est pas d'accord avec le raisonnement, avec les conclusions. Son seul argument consistant était : est-il possible que les membres du gouvernement soient à ce point idiots qu'ils ne comprennent pas cela ? C'est une bonne question, celle que se posent un grand nombre de gens.

Man'kov et Gorškov ne partagent pas les mêmes idées politiques sur l'essentiel, c'est-à-dire sur les conclusions de Man'kov quant à la nature du régime ; ils se disputent sans parvenir à un consensus (fait particulièrement piquant, ils le font dans la salle de cours, en présence de tous les autres élèves qui chuchotent probablement eux-mêmes). Seule la discussion incite Man'kov, nerveux et conscient de son manque de clarté, à développer et à assembler des impressions éparpillées, pour en tirer des conclusions et aboutir à un jugement critique du régime.

Deux points nous semblent cruciaux. D'une part la confiance est requise pour discuter de ces sujets car, en dépit des appels à «signaler» des entretiens

feodal'noj Rossii v kontekste politiki i ideologii (1930-1950-e gg.) [L'historien et le pouvoir: la science historique en URSS et la conception de l'histoire de la Russie féodale dans le contexte de la politique et de l'idéologie (1930-1950)], Brjansk: Izd.vo Brjanskogo gosudarstvennogo universiteta im. Akad. I.G. Petrovskogo, 2005. 
« antisoviétiques », l'intégrité de Gorškov est au-dessus de tout soupçon pour Man'kov. D'autre part, la reconnaissance mutuelle des faits dont ils parlent car bien que leurs points de vue diffèrent - les deux interlocuteurs sont d'accord sur les faits. Sans cette reconnaissance réciproque des faits, sans cette base que sont ces objets partagés sur lesquels on peut prendre appui et se positionner, par rapport auxquels on peut avoir ou assumer un point de vue, entente ou dispute seraient impossibles. Seule cette reconnaissance mutuelle des faits permet d'avoir une prise cognitive sur la réalité extérieure, au moins en partie. Aussi Man'kov est-il pleinement conscient qu'à l'époque stalinienne la reconnaissance des faits ne va pas de soi. À propos des célébrations de l'anniversaire de la révolution d'Octobre, il écrit : « Il faut dire que le gouvernement gaspille de plus en plus de moyens pour ériger une façade, un aspect extérieur favorable, de la vie soviétique. » [10.11.1933]. Il est souvent difficile de voir derrière cette façade, de reconnaître et de rassembler les faits pour restituer une réalité plus « vraie » que la présentation officielle. Man’kov s'en prend au principe même du réalisme socialiste : la réalité n'y est pas décrite telle qu'elle est, mais telle qu'elle devrait être $^{7}$ et comme si elle l'était. Or ce «comme si »n'est jamais clairement signalé. L'état idéal souhaité se donne comme état réel. Et ce « réalisme » n'est pas seulement un style littéraire. La lecture de l'article d'A. Tolstoj sur le canal de la mer Blanche (Belomorkanal) suscite chez Man'kov la réflexion suivante :

30.9.1933. Dans le numéro du soir des Izvestija on a publié un article d'Aleksej Tolstoj, « Le nouveau continent ». C'est un conte, assez bien écrit, sur l'avenir et le présent de notre région du Nord [...] qui est fait de façon tendancieuse et peu consciencieuse, en ce qu'il confond les temps, les faits et les événements ${ }^{8}$ [...]. La commande sociale de forger l'idéologie de la population est ici réalisée très vilainement. Tolstoj écrit : «En 1943 le train électrique avance rapidement le long du lit de la rivière desséchée... [...] Il y a dix ans, en décembre 1933, fut branché le premier générateur de la première centrale électrique Nevstroj ».

Il semble que ce soit Tolstoj lui-même qui ait pris ce train électrique mythique en 1943 [...] : ce n'est déjà plus la toundra morte (elle était morte en 1933), mais [un paysage] plein de vie, creusé de nombreux canaux d'irrigation, ensemencé de blé et de pommes de terre résistantes au froid... Vous mentez, vous mentez cyniquement, Aleksej Tolstoj !

C'est un procédé inventé récemment de parler de l'avenir lointain comme du présent ; et de l'avenir proche et du présent comme du passé ; un moyen tendancieux et spéculatif de confondre les temps, déjà un subterfuge usuel de nos « socialistes », destiné à exercer de l'influence sur la conscience des gens afin de s'en emparer.

Voilà la genèse d'une masse d'illusions qui s'enracinent actuellement dans les esprits.

7. Katerina Clark, The Soviet Novel: History as Ritual, 2. ed., Chicago : Chicago University Press, 1985; SheilaFitzpatrick, The Cultural Front: Power and Culture in Revolutionary Russia, Ithaca, NY : Cornell UniversityPress, 1992, p. 216-237.

8. Les italiques des citations sont toujours miennes, M.G. 
Cette illusion affecte et pénètre la réalité, elle crée une réalité propre précisément parce que les individus s'approprient la représentation officielle, parce qu'ils la font renaître à chaque fois qu'ils en parlent. Qu'il est difficile, même pour un observateur aussi lucide et attentif que Man'kov, de démêler réalité et fiction.

D'où les doutes qui se font jour surtout dans la solitude, lorsqu'à la clarté du dialogue avec autrui se substitue ce dialogue intérieur aux voix multiples, notamment celles des gens que l'on prend particulièrement au sérieux. C'est alors que Man'kov peut inverser sa propre définition de ce qui est « façade » et de ce qui est « contenu ». C'est ce qui se passe après sa critique de la dégradation des conditions de vie et du rôle de l'armée comme exploiteur de la population productive :

Et si tout ce que j'ai écrit n'était pas vrai ? [...] Si ce n'était que la face extérieure des phénomènes, la pure apparence, totalement indispensable, pour ainsi dire légitimée par l'histoire, et si derrière cette apparence se cachait l'essence lumineuse et claire ?! ? Et moi, si je ne me suis pas aperçu de cette essence, parce que je suis un rien, une créature myope, seulement capable de souiller la vérité, mais absolument incapable de la dévoiler? Et du coup Gorškov aurait-il raison ?? Il est peut-être vrai qu'en réalité nos dirigeants [...] désirent vraiment et sincèrement [le bien du peuple], mais sont contraints de faire leur politique par la force de circonstances objectives ?? De l'environnement ennemi, par exemple ? [7.4.1933 ${ }^{9}$

Ne sont pas remis en cause les faits qu'il cite : les prix, les conditions de vie, le statut de l'armée (faits reconnus par Gorškov), mais ses «conclusions », sa condamnation du gouvernement. Il est enclin à admettre, ne serait-ce que provisoirement, l'argument principal de son ami : il est possible que la politique stalinienne, qui fait souffrir le peuple à cause d'un budget militaire excessif, soit justifiée par l'énormité tout à fait réelle de la menace extérieure ${ }^{10}$.

Lors de leur rencontre suivante, Man'kov est très déçu par son ami qui, après avoir obtenu son passeport et s'être « établi comme peintre dans une organisation coopérative », « reçoit un salaire assez bon » et ne vit désormais que pour son amusement. [12.6.1933] La visée du bien commun a disparu de ses propos, il ne voit guère plus loin que son assiette. Man'kov l'accuse d'égoïsme et d'attitudes petites-bourgeoises, d'autant plus qu'il n'est apparemment parvenu à sa (nouvelle)

9. Hellbeck, (Revolution on my Mind, p. 101 et 384, note 74) cite ce même passage et affirme que, comme beaucoup d'autres « passages autocritiques », il manque dans le journal publié. Or ne manque que la dernière phrase (qui semble suivre le passage tel que nous l'avons cité d'après la publication) : «Peut-être y a-t-il véritablement un petit diable jaune décrépit en moi, l'ennemi de classe, comme ils écrivent dans les journaux ?? Est-ce possible ? Est-ce possible ? » Dans sa présentation Hellbeck omet la référence à l'armée comme exploiteur du peuple et coupe dans sa citation aussi bien l'allusion à Gorškov que «l'environnement hostile ». Il manque ainsi une distinction qui nous semble cruciale, entre la vérité des faits et une vérité politico-idéologique. Hellbeck excipe surtout de ce genre de passages pour souligner la fragilité, voire l'inconsistance de la critique en Union soviétique stalinienne.

10. Cette menace de l'ennemi étranger est la source de l'hésitation principale (plus ou moins manifeste) chez la grande majorité des critiques, ce qui est logique puisqu'il s'agit d'un facteur qui est en dehors de leur horizon de perception et par là difficile à évaluer à distance sans sources ou intermédiaires suffisamment fiables. 
« philosophie » que par le biais de l'amélioration de sa situation matérielle. Sa critique antérieure de la politique du régime se réduit maintenant à de purs grognements, particuliers et éphémères, fruits de sa situation personnelle précaire - une perspective en contre-plongée. La métaphore de la bagarre montre bien à quel point Man'kov s'insurge contre l'ami :

Je sens que le fossé de la divergence croît et s'élargit entre nous. Bientôt il atteindra ses dimensions limites [...]. Il faudra alors que l'un de nous deux ait les épaules à terre et que l'autre l'y maintienne en lui mettant un pied sur la poitrine. Plus ce fossé s'agrandit, plus les intervalles entre nos rencontres augmentent.

Mais immédiatement après cet accès de colère, Man'kov relativise son jugement et admet :

12.6.1933. Néanmoins, quand je ne le vois pas pendant longtemps, je suis saisi par une tristesse lourde. Indubitablement ça vient de la solitude - d'une solitude complète et sans issue.

Le meilleur moyen d'objectiver les impressions et les épreuves est de tenir un journal. Le journal est une échappatoire à travers laquelle un sujet qui est en train de s'effacer peut regagner son équilibre d'esprit et du corps.

Il est cependant nécessaire que le journal tombe sur une terre fertile, capable de l'accepter - ou bien de le repousser cruellement et sans pitié. Le premier lecteur de mon journal a été Gorškov.

Nous voyons combien le lien de la communication est étroit entre eux. Passage révélateur dans la mesure où Man'kov dessine, à partir de sa propre expérience, le danger de la personne isolée. Cela correspond tout à fait à ce que Hannah Arendt dira plus tard du sujet totalitaire. La « solitude complète et sans issue » engendre une dépression, une «tristesse lourde » qui risque de vous placer dans un état d'esseulement propice à la soumission de la pensée à une logique totalitaire, purement déductive et impitoyable ${ }^{11}$. Pareille logique s'exprime parfaitement dans les passages «solitaires », comme celui cité, où la critique a tendance à se retourner contre son auteur. Serait-il myope ? Les arbres ne lui cachent-ils pas la forêt ?

Ici Man'kov analyse la fonction de son journal. Il est censé «objectiver ses impressions », c'est-à-dire donner substance au réel qui s'offre à la perception pour permettre de faire face à l'impact de la propagande. Il doit matérialiser le dialogue intérieur et se substituer, ne serait-ce que temporairement, à un interlocuteur extérieur : il s'agit de garder ses perceptions et impressions pour autrui. Man'kov relie explicitement écriture du journal et communication directe. Le journal, offert à l'autre pour être lu et commenté se transforme ainsi potentiellement en lettre destinée à «tomber sur une terre fertile». Il y a donc besoin de trouver des destinataires adéquats et dignes, qui soient en mesure de répondre sérieusement. Une hypothétique

11. Hannah Arendt, Elemente und Ursprünge totalitärer Herrschaft: Antisemitismus, Imperialismus, totale Herrschaft, Munich-Zurich: Piper, 2003 (en français, Les origines du totalitarisme, P. : Seuil, 2003) ; Id., The Life of the Mind, New York : Harcourt Brace Jovanovich, 1981, c. 1978 . 
postériorité, réceptacle vague d'un dialogue imaginaire avec un avenir lointain ${ }^{12}$, ne suffirait pas à cette «objectivation ». Elle ne remplace aucunement l'interlocuteur réel dans l'immédiat (ou dans un horizon temporel proche), c'est-à-dire le face-à-face d'un échange véritable. Dans cette optique, le journal n'est qu'un pis aller : c'est une « échappatoire » (lazejka) à la solitude, qui aide à surmonter ces longs intervalles entre les rencontres et dialogues réels quand il y a possibilité d'aborder et de discuter de propos critiques qui seraient réprimés dans presque tous les autres contextes. Mais il n'aboutit ni à l'autosuffisance ni à l' autonomie du diariste.

Man'kov conçoit son journal comme le dépôt de ses observations et idées critiques, souvent d'une grande généralité, qui portent sur l'ensemble de la société et du système. À l'égard d'un environnement qui clame d'une voix unanime le contraire, ces pensées dissidentes solitaires sont extrêmement fragiles faute d'un appui extérieur. Il faut au moins en faire part quand même elles ne seraient pas partagées sinon (« une terre fertile, capable de l'accepter - ou bien de le repousser cruellement et sans pitié »), prises au sérieux et discutées en tant que points de vue acceptables et légitimes, elles risquent soit de s'effacer dans la demi-obscurité du non-dit, d'une sorte de subconscient refoulé et inaccessible, soit de sombrer en obsessions pathologiques et d'abandonner tout ancrage dans le réel. Tel est le cas du frère de Man'kov, Nikita, qui tombe sérieusement malade au point d'être interné en hôpital psychiatrique en 1938 pour avoir frappé un milicien [7.6.1938]. C'est plutôt une chance pour lui car dans ses moments de crise il peste contre Stalin sans se soucier de l'entourage. Sans aucun doute, Nikita n'a aucune échappatoire, personne à qui il pourrait faire part de ce qui le trouble de façon latente. La gravité de son état n'est remise en cause ni par ses parents ni par son frère. Plus remarquable encore, cela vaut aussi pour ses discours critiques : même dans son journal, Man'kov n'établit jamais de lien entre ses propres propos critiques et ceux de son frère, pourtant fort similaires à en juger par ce qu'il écrit. Il n'y a donc pas d'échange sérieux entre les frères ${ }^{13}$.

Les limites de l'écriture diariste sont parfois exprimées très clairement, comme lorsque Man'kov est sur le point de quitter l'usine Triangle rouge. Bien qu'il en ait assez de son travail, il apprécie ce microcosme, avec ses gens différents et sa plurivocité ${ }^{14}$. Même s’il s'est souvent senti étranger parmi ses collègues, il est particulièrement inquiet lors de son dernier jour à l'usine à la perspective d'un changement pour un nouveau milieu. C'est avec ces gens qu'il connaît de longue date qu'il a pu

12. Cette dimension d'une parole implicitement adressée à un public, apparemment dans un futur non précisé, se trouve, p.ex., dans la première entrée conservée, celle sur la dispute avec Gorškov au cours de la bibliothèque publique : Man'kov y explique entre parenthèses où et quand il fait ses études, renseignements superflus pour lui-même, mais aussi pour Gorškov et d'autres proches (30.3.1933]. Peut-être s'agit-il d'explications rajoutées au moment de la publication du journal?

13. Man'kov semble très réticent à analyser la maladie de son frère. Il ne prend pas au sérieux ses injures précisément parce que ce sont des insultes gratuites, non des jugements équilibrés qui chercheraient à démontrer et à prendre appui dans la réalité. Voir, p. ex., son entrée du 3.2.1940.

14. Voir p. ex. l'entrée du 30.3.1933 et surtout celle du 3.10.1933. 
avoir les conversations les plus ouvertes, comme avec Gorškov depuis l'enfance ${ }^{15}$. Être arraché à son milieu habituel (volontairement ou non) réduit considérablement à l'évidence les interlocuteurs avec lesquels communiquer ouvertement.

18.4.1934. [...] Et peut-être suis-je en train de commettre une grande vilenie : je quitte l'usine, ce lieu qui m'a appris à comprendre la vie. Ici il y a des gens variés, et des affaires variées. Et je pars. Où ? À la bibliothèque. Dans un monde calme et monotone où je ne parlerai qu'avec un groupe très étroit de gens. Qui me dira si mon départ est une vilenie, un crime, ou non ? Pourquoi vous taisezvous? Alors ? Pourquoi ? Vilenie ou non?

Ainsi il cherche ici vainement les multiples voix de son for intérieur, cette ressource à même de prolonger le dialogue extérieur.

La douloureuse pénurie d'interlocuteurs pour ses idées politiques non conformistes l'amène à un acte qui témoigne et de son audace et de son désespoir. Après avoir lu un livre Anatolij K. Vinogradov (1888-1946) ${ }^{16}$ sur l'homme superflu du XIXe siècle, il se met à écrire à l'auteur. Le journal devient ici lettre - ou, plus précisément, brouillon de la lettre qu'il enverra de fait.

Man'kov ne semble redouter ni la censure (perlustracija) ni une dénonciation éventuelle de Vinogradov. L'écriture littéraire lui semble comme le miroir de l'âme et de la pensée la plus profonde de cet auteur - et cela en dépit, ou plutôt en raison, des « quelque trente lignes » consacrées à la fin du livre à l'époque contemporaine. Il y note une rupture stylistique éclatante, qu'il qualifie de subversion et y décèle un signal d'opposition qui s'adresse à des lecteurs sensibles et critiques - des gens comme lui ? L'action et les attentes de Man'kov s'inscrivent ici dans un ethos hérité de l'intelligentsia progressiste du XIX ${ }^{\mathrm{e}}$ siècle, une intelligentsia engagée, orientée vers l'avenir, ne craignant pas le sacrifice personnel ${ }^{17}$. Dans sa lettre Man'kov aborde cette question par le biais de l'idée de l'homme superflu. Si dans son livre ${ }^{18}$ Vinogradov met l'accent sur l'homme superflu « apatride » (neprikajannyj) qui vit dans le passé, tout en refusant la réalité telle qu'elle est, Man'kov souligne qu'il y a encore un second type d'homme superflu, celui qui non plus n'accepte pas le présent, mais au nom de l'idéal d'un avenir meilleur, non pas pour «se complaire dans le passé ». C'est le type du «révolutionnaire ». Le premier type serait totalement superflu, le

15. Ce sont aussi ses amis d'enfance de Kašin qui lui racontent les cruautés qu'ils ont vues dans les villages pendant la collectivisation. Dans sa critique de la politique économique et sociale, Man'kov s'appuie largement sur ces témoignages individuels qui rompent avec le tabou de la propagande. Voir l'entrée du 7.10.1933.

16. Apparemment, il s'agit de la série Histoire du jeune homme du XIXe siècle que Vinogradov a publiée en collaboration avec Gor'kij. Voir l'aperçu biographique sur http://www.desnalib.ru/ index .php?parent=132\&pre=118, consulté le 30.3.2007.

17. Voir Nina Iosifovna Belunova, Družeskie pis'ma tvorčeskoj intelligencii konca XIX nacala $X X$ veka. (Žanr i tekst pisem) [Lettres amicales de l'intelligentsia créatrice de la fin du XIX ${ }^{\mathrm{e}}$ - début du XX $\mathrm{X}^{\mathrm{e}}$ siècles, (genre et texte de lettres)], SPb. : Naučnoe izd., 2000, notamment sur la tradition de la lettre dans les milieux de l'intelligentsia du XIX ${ }^{\mathrm{e}}$ siècle.

18. Il n'est toutefois pas clair de quel livre de Vinogradov il est question. 
deuxième ne le serait que « sous condition, relativement », c'est-à-dire en fonction du contexte systémique et social. Sans fard, Man’kov pose ses questions hérétiques :

Quel genre de gens superflus peut-il y avoir et y en a-t-il en effet dans nos conditions actuelles ? Vous affirmez gratuitement qu'il n'y a pas de gens superflus chez nous $[\ldots]$ : «l'homme sain, fort, et solidaire avec le reste de l'organisme social, vit et meurt joyeusement après avoir terminé le cycle de ses liens vitaux et créateurs, cet homme a remplacé le héros typique de la période du capitalisme » [...]. À mon avis on trouve et l'un et l'autre type d'homme superflu dans notre réalité, car y a lieu encore une querelle sociale atroce. Le fait qu'ils ne soient pas encore reflétés dans la littérature est dû à des raisons particulières.

Dites-moi, comment un jeune homme peut-il être «solidaire avec l'organisme social » si, à l'intérieur de cet organisme règne la poigne de fer du mensonge, de la contrainte et de l'arbitraire, si presque tous sont pris par la production (pas tous, il est vrai, quand même et surtout, pas à des conditions égales, et de loin), alors que sur le plan de la consommation très peu de gens y participent, et encore dans leur majorité ceux qui ne sont pas immédiatement impliqués dans une activité productrice : les membres du parti occupant des postes de commandement, les fonctionnaires, l'armée, la GPU, etc. ??! N'est-ce pas à l'image du passé, lorsque des milliards étaient gaspillés pour la bande des ministres [...] ? !! Dites-moi, un jeune homme peut-il être solidaire d'une réalité où les gens dans les districts les plus riches en blé ont le ventre qui enflent et meurent de faim, $[\ldots]$ et dans les villes $[\ldots]$ sont licenciés et jetés à la rue $[\ldots]$ pour un seul retard de plus d'une heure, où ils sont privés de la ration de chien misérable qu'ils avaient reçue pour leur travail dans l'entreprise ?!

Peut-il l'être [solidaire], Anatolij Kornil'evič ? Ou bien ne doit-il pas plutôt emprunter la voie de la négation de cette réalité, par principe et jusques aux racines. Je ne parle pas du premier type de l'homme superflu dans un sens absolu [...]. Mais le type du jeune homme superflu dans un sens relatif, bien entendu celui qui est fort et intransigeant, n'est-il pas particulièrement réel et palpable de nos jours ? Et s'il est aujourd'hui encore caché dans les tréfonds de notre pays, cela ne veut pas dire qu'il ne se fera entendre demain ou après-demain. Si vous le trouvez nécessaire, répondez-moi ! [...] Je vous en serai reconnaissant. Seulement, je vous prie instamment d'écrire dans votre propre langue et avec vos propres mots, et non pas dans le tam-tam des journaux, qui est un grand mensonge du début à la fin, et où l'on s'égare irrémédiablement. [31.7.1933]

Que dire d'une telle lettre, envoyée en l'an 1933 à un auteur qu'on n'a jamais rencontré ? Est-elle audacieuse ou tout simplement folle ? Man'kov peint l'image d'une société en état de fermentation sous-jacente : il a non seulement l'impression qu'il y a beaucoup de mécontents, mais qu'il y a, malgré la liquidation des derniers groupuscules d'opposition, encore beaucoup de vrais révolutionnaires qui ont gardé leurs idéaux et seraient même prêts à lutter contre le régime pour la réalisation de leurs rêves. Sa lettre est un appel à la résistance, ce qui n'est possible qu'en s'associant, en agissant de façon concertée. Animé de l'élan révolutionnaire qu'il oppose à la réalité mauvaise de l'exploitation et du «mensonge », Man'kov est prêt à sacrifier sa vie pour la cause, comme l'étaient les révolutionnaires de 1917. À l'égard du risque encouru par l'engagement révolutionnaire, Man'kov suppose que des gens convaincus sont prêts à agir et à aller jusqu'au bout - même contre le régime soviétique si nécessaire, c'est-à-dire s'ils le reconnaissent comme exploiteur et injuste. 
Ainsi, tout en inversant la logique des procès-spectacles de la première moitié des années 1930 contre les spécialistes bourgeois, Man’kov voit dans les « saboteurs ${ }^{19}$ de tels hommes superflus révolutionnaires et les approuve. Il admet ce dont on les accuse, mais y voit des actes de résistance hérö̈ques, révolutionnaires et progressistes. Aussi s'insurge-t-il contre la tentative du régime de faire croire au peuple qu'il s'agit de manifestations isolées, alors que selon lui c'est un phénomène de masse, bien qu'encore réprimé.

20.4.1933 [...] Curieux et pitoyable est encore cet effort de présenter ce procès comme un procès isolé d'un groupe d'intellectuels dégénérés, de criminels issus du camp des fonctionnaires. Que fait-on alors de Zivert et Lebedev ??? Le premier - un « serrurier avec une éducation primaire (s nisšem obrazovaniem) », le deuxième - un contremaître (master). Ne seraient-ils pas, par hasard, issus des couches ouvrières?

Une fois de plus le procès a montré que le mécontentement des masses croît rapidement, que même s'il reste jusqu'ici sous-jacent, il remonte par endroits à la surface, avec force, ce qui est un fait éloquent en $\operatorname{soi}^{20}$.

Mais comment agir pour échapper à l'oblomovisme, à l'inactivité caractéristique de «l'homme superflu » qui « vit dans le passé »? Les rares entretiens critiques avec Gorškov sont peut-être à même de rendre plus claires les idées de Man’kov, mais ils sont loin de mobiliser les masses mécontentes. La lettre à Vinogradov restera sans réponse. Étant donné que pour Man'kov le problème principal réside dans les « mensonges » du régime, dans la façade que celui-ci érige pour tromper la population sur la réalité soviétique, sa tâche principale, et celle de tout révolutionnaire véritable, est selon lui d'éclairer le peuple sur cette réalité. Il est bien conscient que sous un régime qui a ses «tentacules » partout, on ne peut plus simplement «aller au peuple» pour l'«éclairer», comme l'ont fait les populistes au XIXe siècle. Aussi l'instruction lui semble-t-elle le chemin le plus prometteur et, à plusieurs reprises, il postule des postes d'enseignement, aussi bien à Leningrad qu'en province, mais ce ne sera qu'au moment où il fera des études à l'université qu'on accédera à ses demandes. Jusque-là il misait sur la littérature, en s'appropriant encore une fois en l'inversant la prémisse stalinienne des écrivains comme ingénieurs des âmes ${ }^{21}$. Il voit les dégâts causés par un Tolstoj et le réalisme socialiste, mais n'en place pas moins son espoir dans des auteurs tenus pour subversifs,

19. Sur le procès de Metro-Vickers et ses conséquences pour la diplomatie internationale, notamment pour les relations soviéto-britanniques, voir Gordon W. Morrell, Britain Confronts the Stalin Revolution: Anglo-Soviet Relations in the Metro-Vickers's Crisis, Waterloo, Ontario :Wilfrid Laurier University Press, 1995.

20. En octobre 1934, lorsque dans les journaux paraît « un commentaire timide sur une nouvelle organisation contre-révolutionnaire », où des ingénieurs « ont diffusé des feuillets » et « trois ont été fusillés », il s'exclame : « En tout cas, gardons un bon souvenir d'eux. Immortalisons-les dans nos cœurs et dans notre mémoire. » [17.10.1934]

21. Voir surtout ses commentaires approbateurs sur le discours de Buharin sur la poésie qui «a hissé sur le pavois la lutte pour une culture authentique et véritable de l'humanité » lors du Premier congrès des écrivains soviétiques. Entrée du 1.9.1934. 
comme Vinogradov. Surtout, il écrit lui-même des contes, rejoint des cercles littéraires afin d'échanger et faire circuler ses idées. Mais son projet de mettre sens dessus dessous l'intrigue du Bildungsroman socialiste (progression de l'être humain «de l'obscurité vers la lumière de la conscience») afin de distinguer l'obscurité et l'implication dans les mensonges du régime de la lumière et de la lutte révolutionnaire contre ces mensonges et contre ceux qui en sont responsables ${ }^{22}$, se révèle irréalisable, même en usant de subtilité. Ses timides tentatives littéraires sont soit rejetées, soit en butte à l'incompréhension (réelle ou prétendue). Il faudra le samizdat pour échapper à la production littéraire « pour le tiroir »...23

\section{Nouvelle constellation des rapports personnels à la veille de la guerre}

Il est peu étonnant qu'en 1938, lorsque le journal reprend, l'ambiance générale ait considérablement changé : la Terreur a encore donné matière à la réflexion de Man'kov sur la nature du régime ${ }^{24}$. Dans le même temps, le diariste est déjà immergé dans un milieu universitaire professionnel d'historiens, qu'il voit toujours d'un œil très critique, mais où il a apparemment aussi trouvé des âmes sœurs.

Un leitmotiv majeur de ses observations après le (léger) recul de la Terreur est le constat du nouveau cynisme du régime. La comparaison avec le nazisme y joue un rôle important. Non seulement dans l'historiographie (son domaine), dans le cinéma et les arts Man'kov note le renouveau du nationalisme, mais aussi dans le traitement quotidien des citoyens soviétiques non russes par les représentants du système. Lorsque pendant la perquisition de l'appartement de sa cousine Zoja, un fonctionnaire du NKVD, « apparemment honnête », lui fait entendre qu'en tant que Russe elle n'aurait rien à craindre, il commente : "Hitler peut se réjouir de sa victoire » [23.9.1938]. Ceci se confirme après le pacte de non-agression germanosoviétique. La création de l'Administration centrale pour la réserve de travail ${ }^{25}$ et surtout les guerres expansionnistes dans les pays Baltes et en Finlande suscitent chez Man’kov des commentaires de ce genre.

Le cynisme du régime va de pair avec un nouveau cynisme des gens de l'entourage de Man'kov face à la réalité soviétique. En seraient affectés de plus en plus, même « les orthodoxes ». Ainsi, un camarade d'études lui parle de ses perspectives professionnelles : il aurait « déjà craint d'évoquer le doctorat (bojalsja zaiknut'sja aspiranturu) » :

- Il y a tant de candidats [doctorants], et encore si abscons, se désespère-t-il en souriant avec sarcasme.

22. Voir le développement de l'intrigue d'un roman projeté. Entrée du 11.10.1933.

23. Cf., p. ex., les contes de Lidija Čukovskaja Spusk pod vodu [Descente sous l'eau] et Sofija Petrovna, écrits dans les années 1930 et 1940, mais qu'elle n'a pu faire circuler qu'en samizdat.

24. Sa famille élargie n'a pas été épargnée. Ainsi sa cousine Zoja est exilée après l'arrestation de son mari. Voir entrées du 31.5.1938 et du 23.9.1938.

25. Glavnoe upravlenie po trudovym rezervam, entrée du 4.10.1940. 
J'ai compris avec surprise que cet homme lui aussi cherche déjà la petite bête. Et moi, j'avais pensé que c'était un orthodoxe et une âme encore limpide. $\mathrm{Au}$ demeurant, maintenant il y en a très peu. Il faut vraiment être un idiot fini pour devenir un orthodoxe. [10.3.1940]

Le fiasco de la guerre de Finlande - et la propagande soviétique qui a parlé d'une lutte révolutionnaire du prolétariat finlandais sous la direction de «son » Gouvernement populaire (gouvernement mis en place par l'URSS en réalité) - semble provoquer des réactions narquoises à la faculté d'histoire :

13.3.1940. La paix. Des petits sourires ininterrompus au département d'histoire. Que se cache-t-il derrière ? De la joie ? Ou plutôt des railleries ironiques ? Tout le monde a la même question sur les lèvres :

- Et qu'est-il devenu le Gouvernement populaire, hein?

Dans les journaux pas un seul mot là-dessus. D'ailleurs, il serait ridicule de parler de l'inexistant et de l'irréalisable, d'une pure fiction. [...] Une chose est évidente : la guerre, même si elle a été gagnée stratégiquement au prix de terribles pertes, a été perdue politiquement de façon ignominieuse. Il y a des rumeurs selon lesquelles la paix aurait été conclue sous la pression de l'Allemagne qui a besoin d'arrières paisibles. Si c'est vrai, cela veut dire que nous sommes influencés par des intérêts étrangers. Or une paix à de pareilles conditions aurait été possible bien avant.

Malgré la difficulté à deviner ce que les gens pensent réellement, Man'kov remarque une sorte de connivence dans les comportements et dans les regards. Il s'agit d'une communication non verbale, qui reste, certes, toujours ambiguë. À propos de comportements tacites semblables pendant la dictature militaire en Argentine, Guillermo O’Donnell ${ }^{26}$ a appelé « voix oblique » cette forme particulière de la « voix horizontale », c'est-à-dire de la communication entre sujets. Selon ce sociologue, "parfois des regards rapides dans la rue ou dans d'autres espaces publics » sont un moyen « dans ce domaine fertile de l'imagination humaine » de se reconnaître comme opposant au régime. À travers la voix oblique «on obtenait cette gratification cognitive cruciale d'une identité collective partagée, ainsi que le bénéfice émotionnel non moins crucial d'affirmer le respect de soi-même en tant que non 'idiotes' ${ }^{27}$. Bien que le non-explicite de ces gestes et signes ne parvienne jamais à la clarté et l'explicite du langage, cette voix (tacite) peut toujours clamer son innocence face aux « orthodoxes limpides ». Le sentiment que ceux-ci sont désormais en minorité est nouvelle et renvoie à un changement d'atmosphère significatif par rapport à celle qui régnait à la veille de l'assassinat de Kirov.

26. Guillermo A. O’Donnell, Counterpoints: Selected Essays on Authoritarianism and Democratization, Notre Dame, IN : University of Notre Dame Press, 1999.

27. O’Donnell emploie ici le terme grec <idiotes>, terme opposé aux <polites $>$. Il s'agit donc de ceux qui se sont soustraits volontairement ou par force (des ostracisés, p. ex.) à la vie de la polis, communauté politique du débat commun des choses publiques. Dans le présent contexte, nous reprenons le terme au sens grec, dans la mesure où il souligne bien l'aspect central de l'isolement. 
Si pour les Argentins dissidents c'est là « un dernier résidu minuscule », rétrécissement extrême de leur persona politique, pour Man'kov elle signifie un gain en termes d'assurance et de respect de soi, du moins par rapport à ce qu'il a expérimenté jusque-là : il ne se sent plus seul désormais avec ses regards «non orthodoxes ». Les signes d'une distanciation du discours officiel dotent ses réflexions d'une nouvelle légitimité. Pourtant, si en pratiquant la voix oblique, les Argentins signalent l'un à l'autre une «préoccupation politique » (en dépit de la «privatisation » patente de leurs vies) et opèrent ainsi, virtuellement en quelque sorte, un agrandissement de leur identité du plus personnel au plus politique, il en est autre pour les étudiants de l'entourage de Man'kov. Habitués à une existence ostentatoirement politisée, ils ridiculisent plutôt l'aplomb de la propagande, à laquelle ils participent eux-mêmes. Ils réduisent par là radicalement l'unanimité réclamée par le régime - et par eux-mêmes. Implicitement ils se « dégonflent », infirmant ainsi l'authenticité de leur propre existence publique, la dénonçant comme un masque, étranger et imposé.

Quels sont les signes par lesquels la réduction est opérée ? Man'kov le dit clairement : plus que tout autre, ce sont les «petits sourires » moqueurs et ironiques qui minent le sérieux d'une grandiloquente rhétorique officielle. C'est à la manière de la constatation que le Roi est nu : il suffit qu'un seul le clame, pour qu'aussitôt tous osent en croire leurs yeux et s'esclaffent de rire. Une fois reconnu comme être humain ordinaire, dans sa ridicule nudité, il perd toute grandeur et majesté royales. L' « abaissement du Roi (de Stalin ou du système) élève ses sujets. Mais dans le cas des « voix obliques » au département d'histoire de Man'kov, tout se passe discrètement. Le soulagement libérateur du rire sonore et sans équivoque ne vient pas, tout se passe à un niveau vague et flou. La «mise à nu » implicite reste extrêmement fragile sans cette verbalisation que serait une véritable «voix» horizontale. Man'kov cherche l'explicite, entre autres par le biais de la provocation :

15.3.1940. On a demandé au chargé de cours B., un type servile avec des yeux de poisson crevé (officioza s glazami dohloj ryby), quel est maintenant le destin du Gouvernement populaire. Il a esquivé la question, mais il s'en est donné à cœur joie sur le fait qu'on a atteint avec brio le but envisagé - protéger Leningrad. C'est la rengaine de n'importe quel journal.

Man'kov sent que «tout le monde a la question sur les lèvres », mais que personne ne la pose à haute voix. Il le fait, sachant qu'il enfreint un tabou, instauré de longue date et subtilement par la presse qui, à partir du moment de la trêve et des célébrations des «succès » de l'Armée rouge «glorieuse », a évité toute référence au «Gouvernement populaire » finlandais de Kuusinen (qui a été sacrifié pour la trêve par Stalin). Les journaux n'ont pas besoin de répondre aux questions qui s'imposent (sans être posées), mais lors du face-à-face la réponse stupide de ce chargé de cours donne satisfaction à Mańkov. Son verdict est confirmé, aussi bien sur le fonctionnement du non-dit que sur les « idiots finis » qui se contentent de répéter comme des perroquets les clichés de la propagande. 
Néanmoins, de véritables entretiens politiques sont beaucoup plus importants pour Man'kov. À la différence de la situation en 1933-1934, il trouve des interlocuteurs à l'université, grâce en partie au nouveau milieu qu'il fréquente, mais pas seulement. Dans ses réflexions critiques il part souvent de comparaisons historiques qu'il souhaite discuter avec d'autres historiens; cela se passe sans doute à l'écart des séminaires et des exposés dans des cadres officiels. Ainsi il établit un lien entre propagande soviétique et divulgation d'histoires de miracles dans le cadre de la politique religieuse prérévolutionnaire [15.8.1938]. Ailleurs il compare l'élan libérateur et l'initiative du peuple pendant le Temps des Troubles avec des phénomènes semblables pendant les révolutions du $\mathrm{XX}^{\mathrm{e}}$ siècle. Dans les deux cas il constate la réaction consécutive : nouveau servage, nouvelles chaînes forgées par le nouveau régime issu du chaos ${ }^{28}$.

Sa principale interlocutrice est K., jeune doctorante qui travaille aussi sur le Temps des Troubles et qu'il décrit comme très intelligente, aux intérêts multiples. Leurs entretiens partent souvent de discussions professionnelles. Un de leurs sujets de prédilection est le travail scientifique, son manque d'originalité, et la pauvreté méthodologique dont il souffre du fait du climat politique répressif et de la pression idéologique :

24.1.1940. [...] Comme toujours nous avions un entretien vif [...].

- Vous savez, Arkadij, nous avons besoin de théorie. Sinon, comme des aveugles, nous avancerons à tâtons dans la science.

- Oui, c'est vrai, je l'interromps, à tâtons dès que nous prenons les choses en mains, mais nous préférons généralement nous accrocher à des autorités. Prenez L'Europe à l'époque de... de Tarle ${ }^{29}$ - c'est le livre d'un penseur génial et indépendant, pour qui il n'y a pas d'autorités : tout est matière à réflexion. Et nous ? Même un petit article est truffé de citations de Marx et Lenin, non, pas une seule pensée vivante et originale $[\ldots]$

— Oui, tout à fait [...] Prenez Solov'ev [...] Un hégélien. Et son cours entre dans un cadre hégélien. Mais où y a-t-il la moindre référence à Hegel [...] Nous ne deviendrons jamais des grands savants [...]

- Même mauvais, mais l'essentiel est de l'être [des savants].

Mais ils parlent aussi plus directement des événements contemporains :

3.7.1940. J'ai rencontré K. Elle ressent avec sollicitude et de manière aiguë tout ce qui est commis dans ce monde. Infatigablement ce feu flamboie en elle et veut en sortir, brûlant sa langue et ses lèvres.

Sa passion dans la discussion de ces questions et sa compassion ardente à l'égard de tout ce qui la heurte profondément et sincèrement, sont véritablement merveilleuses. Elle a besoin d'un interlocuteur et d'un auditeur. Elle parle beaucoup et avec ardeur, et souvent il est même difficile de l'interrompre pour placer ses propres remarques! Sur le fond de la masse des étudiants, profondément indifférents et sans personnalité, seulement préoccupés de leurs petites affaires

28. Voir entrée du 26.2.1941.

29. Important livre de Tarle (1875-1955) sur l'impérialisme, publié en 1927 et conçu comme une préhistoire de la Première Guerre mondiale. 
personnelles, cette fille, avec son âme insoumise révoltée et sa pensée tourmentée, est un phénomène lumineux et joyeux.

Bien que T., une autre étudiante, ne réponde pas vraiment aux avances amoureuses de Man'kov, ils n'évitent pas non plus les sujets politiques. Ils évoquent ainsi Amusin, un étudiant qui, à l'apogée de la Terreur, a été accusé lors d'une réunion universitaire d'avoir organisé et dirigé un cercle antisoviétique, puis arrêté :

nous étions tous les deux persuadés qu'il n'y survivrait pas [...] Nous avons parlé de son destin, injuste et cruel, à quel point on l'avait traité de manière grossière, indélicate et cruelle à la faculté, et ma chère et aimable T., toute emportée, s'est même écriée : «Quels salauds ! » [30.12.1939]

On peut supposer que bien d'autres réflexions critiques des pages du journal de cette période sont nées de tels entretiens et en présentent en quelque sorte le prolongement, par mutation du dialogue avec autrui en un dialogue intérieur. Ces conversations intenses ont des répercussions sur ses lectures, et pas seulement sur leur choix : plus nettement qu'en 1933-1934, Man'kov conçoit ses lectures insatiables (historiques et littéraires) comme une forme particulière d'échange avec le texte. «Quand je lis quelque chose de bien, je lis deux textes à la fois : celui qui est devant moi, et un autre, imaginé par moi-même, avec lequel je complète, continue, et même modifie, le texte qui n'est pas le mien. » [16.7.1940]. Pareil dialogue ne va pas de soi : la pensée totalitaire déductive, telle que l'a conceptualisée Hannah Arendt et telle que nous pouvons l'entrevoir dans nombre d'ego-documents de l'époque ${ }^{30}$, ne supporte plus ce dialogue et le pluralisme qu'il implique, tous deux étant douloureusement appréhendés comme un dédoublement pathologique de la personnalité. La configuration doute versus mise en cause de son propre jugement critique, telle qu'on peut la repérer dans certains passages du journal de Man'kov de la première moitié des années 1930 - configuration qui contient le germe d'une telle appréhension - disparaît de ses notes de 19381941. Voici comment il explicite ce dialogue intérieur :

8.9.1940. Cette nuit j'ai eu une conversation avec mon esprit, c'est-à-dire avec moi-même.

- Tu dois, me dit-il, rassembler toute ton énergie et tes forces que tu as jusqu'ici gaspillées de façon tellement mesquine et sale, et les concentrer dans la direction que tu as choisie. Tu dois accomplir quelque chose qui te permettra de t'élever au-dessus des ruines de ta propre existence.

J'ai donné mon accord.

La voie que son « esprit » l'encourage à emprunter est celle de la grandeur éthique : dépasser une existence dédoublée entre théorie (critique) et pratique (inactive). « Changer la réalité au lieu de la contempler» était le mot d'ordre, tout à fait marxien, du «type relatif» de l'homme superflu [31.7.1933]. Apparemment 
Man'kov pense à une confrontation avec la falsification de l'histoire - numéro de haute voltige dans l'environnement stalinien.

C'est d'autre part l'impression que les canaux informels d'information sont moins anonymes qu'auparavant. Certes, beaucoup de « renseignements » arrivent toujours sous forme de rumeur, par exemple sur cet étudiant enlevé de force qui avait déclaré publiquement que la loi sur la suppression des bourses et l'enseignement payant était une violation de la Constitution de 1936 et que le Sovnarkom n'avait pas le droit de la faire passer. On l'aurait passé au crible dans un appartement privé en le persuadant qu'il s'était trompé, pour finalement le relâcher en l'exhortant à ne souffler mot de l'incident. À sa demande d'être ramené à la maison de la même manière qu'on l'avait emmené (en voiture), ses ravisseurs auraient poliment refusé. Man'kov note cette histoire sous la rubrique «Entendu quelque part » pour conclure : « je pense quand même que c'est une blague » [10.10.1940]. Apparemment l'audace de cet étudiant et la clémence de ses détracteurs lui paraissent bien trop invraisemblables. De sa tante Man’kov apprend « qu'il y aurait eu (c'est B. Man'kov, mon cousin, qui lui a raconté) une mutinerie dans la prison où il [B. Man'kov] avait récemment travaillé. Soixante détenus s'y seraient insurgés. On a tiré sur eux à la mitrailleuse. Seize ne se sont pas relevés. » Man'kov reste un peu sceptique, dans la mesure où son cousin n'y travaille plus et n'a pas pu voir de ses propres yeux ce qui s'y était passé : « Je note ça comme une rumeur, mais j'admets que c'est tout à fait possible.»[2.6.1938]. «Une étudiante » avec qui Man'kov entretient apparemment des rapports beaucoup moins proches, et dont le mari est médecin au front en Finlande, lui parle d'une grève de la faim des prisonniers de guerre finnois, ce qui contredit de façon éclatante les « informations » données par les journaux sur l'enthousiasme de la population pour les « libérateurs » soviétiques [11.12.1939]. Ce que Man'kov apprend via K. et son ami L. sur la soviétisation des pays Baltes relève du même niveau de fiabilité. Mais se révèle encore une autre dimension qui dépasse le cadre de ce qui se propage de bouche à oreille : il s'agit de documents écrits qui commencent à circuler :

[K.] m'a donné un conte de L. sur l'Estonie. Là-bas on s'habille avec soin [...]. Dans les rues et sur les places de Tallin on gare les vélos sans qu'il faille les surveiller. Il y a énormément de marchandise [...]. Le peuple [...] ne sait presque rien sur les bolcheviks. De Smetona ${ }^{31}$ qui s'est enfui, on dit : « Nous avons eu un bon président. C'est dommage qu'il lui soit arrivé un tel malheur».

[...] Le paysan pauvre (bednjak), chez qui habite L. et qui a deux chevaux, trois vaches et encore quelques bricoles, n'a absolument pas compris pourquoi on voulait lui attribuer de la terre confisquée, qui appartenait jusqu'alors au propriétaire foncier. Le peuple vit dans l'aisance, tranquillement et calmement. Se pose seulement la question, pourquoi fallait-il que nous nous y immiscions avec notre libération? Non pas pour les libérer de leur vie dans l'aisance et dans le calme mais pour leur apporter nos infirmités, notre misère et notre cruauté ? [5.10.1940]

31. Antanas Smetona (1874-1944), président de la république unie des pays Baltes au moment de la conclusion du pacte de non-agression, prônait la résistance armée contre l'annexion soviétique. 
Sans trop aimer L., Man'kov est impressionné par le courage d'écrire un conte sur son expérience estonienne, conte qui contredit radicalement la propagande soviétique sur les peuples baltes opprimés et qui soulève précisément les «questions » critiques fondamentales que Man'kov pose dans son journal. Apparemment L. a écrit ce conte pour le faire passer de mains en mains sans trop se soucier de dissimuler son nom d'auteur. L. fait confiance à son amie K., mais aussi aux personnes à qui elle décidera de transmettre son manuscrit. N'est-ce pas là déjà un précurseur du samizdat ? Cette recherche d'une sorte de contre-public ne témoigne-t-elle pas de changements majeurs dans les comportements et notamment dans les rapports de confiance, au moins dans le milieu universitaire fréquenté par Man'kov ? Ces expériences ne vont-elles pas à l'encontre de ses conclusions sur la société et la famille «désagrégées en atomes » et sur l'Homo homini lupus est régnant? [4.11.1940]. Le fait qu'il est capable d'envisager à vue d'oiseau les phénomènes, ne témoigne-t-il pas de ce qu'il est (déjà) moins isolé que son image d'une société atomisée ne le laisse croire ?

Man'kov décrit même des manifestations collectives qui dépassent considérablement le niveau des vagues sourires ironiques évoqués à l'annonce de la paix avec la Finlande. La voix oblique prend forme, elle ne marque plus seulement une vague connivence entre personnes qui semblent s'accorder dans leur scepticisme à l'égard des proclamations de la propagande, mais aussi une certaine solidarité face aux autorités. Ainsi, lorsqu'à la chaire d'histoire il y a plusieurs exposés sur « Stalin et les mouvements paysans à l'époque du féodalisme », la réunion se passe « de façon officielle et lugubre. Aucune réaction aux exposés. On a écouté et on s'est dispersé » [22.2.1940]. Outre ce genre de résistance passive, il semble également y avoir des manifestations collectives plus actives, telles que la soutenance de thèse (doktorskaja dissertacija) du médiéviste et spécialiste des chroniques Mihail D. Priselkov (1881-1941) qui a passé des années aux Solovki ${ }^{32}$ et qui est vu comme un symbole de l'intégrité de la science face à l'emprise politique omniprésente. Les historiens semblent unanimes en attribuant une signification politique majeure à l'événement :

17.6.1939. M.D. Priselkov a soutenu sa thèse de doctorat. C'est devenu toute une manifestation. Beaucoup de gens sont venus, même de l'extérieur... Luimême [...] a prononcé le discours inaugural, épatant par sa force, sa vérité, sa profondeur, sa chaleur et son style.

On a commencé à lire son autobiographie, mais il est peu probable qu'il y ait quelqu'un dans l'auditoire qui ne connaisse pas sa biographie mieux et plus profondément que ça, et on savait parfaitement ce qui était sciemment passé sous silence dans le texte [...], à savoir que sous le pouvoir soviétique il avait été emprisonné aux îles Solovki pendant 8 ans avec Platonov. [...]Après il y eut allocutions, congratulations, cadeaux... De notre cercle nous lui avons apporté

32. M.D. Priselkov fut arrêté en 1931 dans le contexte de l' " affaire académique », accusé avec de nombreux autres savants d'avoir fondé l'Union nationale de la lutte pour la renaissance d'une Russie libre. Voir http://www.encspb.ru/ru/article.php?kod=2804021760, consulté le 25.4.2007. 
un coûteux bouquet de fleurs. [...] Tout le monde était très excité, la salle a éclaté d'applaudissements [...] M.D. pleurait.

C'était une heure du triomphe de la science, de l'humanité et de la vérité.

Man'kov se rend compte que les représentants du régime craignent de plus en plus cette résistance passive, un symptôme parmi d'autres indiquant que les appuis du système s'amenuisent et sont en train de se dissoudre. À en croire les observations du diariste, les mesures (et menaces) du régime pour conserver une « façade » (de plus en plus effritée) perdent de l'efficacité, à l'occasion des célébrations du $23^{\mathrm{e}}$ anniversaire de la révolution d'Octobre, par exemple :

5.11.1940 Bien longtemps avant on a commencé l'agitation pour que tout le monde vienne à la manifestation et à la grande assemblée solennelle. Il fallait même qu'on signe. À l'une des réunions, sur l'appel ardent de la tante du parti Motja (part-t'ti Moti) de prendre part active à l'assemblée, quelqu'un a demandé en quoi [cette participation active] consisterait. La réponse : «En étant présent ».

Ils craignent que le peuple n'aille les trahir, ne vienne pas, qu'il ait perdu la foi $[. .$.$] Ils ont peur et cherchent à tout prix à soulever et réveiller l'esprit tita-$ nesque endormi.

Puis, deux jours plus tard, suit le résumé de la manifestation : «Nous avons tous signé la veille que nous viendrions à la manifestation. Une minorité est allée. Maintenant c'est une tactique : promettre n'importe quoi oralement et même par écrit » [7.11.1940].

Toutefois, ce n'est pas l'éclat de rire sur le Roi nu qui est à même de balayer un système inhumain : les petits espaces «libres » résultent du fait que les sujets apprennent à réagir aux «mensonges » en mentant aussi. Ainsi un camarade de Man'kov s'est laissé « séduire par une bourse de 1000 roubles » [24.1.1940] et a «vendu son âme au diable » [31.1.1940] en enseignant l'économie politique à l'Académie de la Marine :

Miša porte un uniforme de la marine. Il lui va bien. Mais il y a maintenant chez lui quelque chose d'obséquieux et de servile [...] Même si à part ça, il est devenu plus charmant et a l'air plus frais. Voilà à quel point il fut tourmenté par la misère. On a parlé de la Finlande [i.e. de la guerre]. Miša était circonspect et fermé » [24.1.1940].

Pour Man'kov, le «marxisme-léninisme » ou l' «économie politique », tels qu'ils sont enseignés en Union soviétique sont une «science à mille et une marottes sèche, lourde et dogmatique », ayant la fonction de «lunettes qui, du fait de leur propriétés optiques, s'adaptent à n'importe quels yeux et sont censées aider à percevoir la 'réalité' » [24.5.1940]. Les compromissions deviennent de plus en plus apparentes. Rares sont ceux qui agissent par conviction, mais les «lunettes » du marxisme-léninisme les aident à couvrir cette vérité douloureuse pour flatter le respect de soi. Man'kov lui-même se reproche de trahir ses convictions, comme lorsqu'il s'est laissé persuader de faire un discours à l'occasion des célébrations de l'anniversaire de la révolution d'Octobre : 
6.11.1938. Sur la soirée au département d'histoire [...]. Le président P., un brun maladroit, me demandait tout le temps de parler au nom des étudiants. J'ai longtemps refusé, mais finalement j'ai cédé. J'ai dit quelques mots sur l'intelligentsia, sur le Manuel [le Précis d'histoire] « qui mérite d'être appelé le meilleur cadeau aux travailleurs à l'occasion du $21^{\mathrm{e}}$ anniversaire [de la révolution] », en conclusion j'ai appelé les gens à participer aux cercles et à préparer des travaux scientifiques. Et tout de suite je me suis rendu compte que ce discours stupide et malhabile pèse sur ma conscience comme une pierre ${ }^{33}$.

Man'kov comprend pourtant que pour la grande majorité des gens de son entourage cet écart ne pose (plus ?) aucun problème : tout en souriant des absurdités de la propagande, ils se sont accommodés d'une vie que Man'kov qualifie de «mesquine ». Ils ne se préoccupent que « de leurs petites affaires personnelles" et, dans leur « indifférence profonde », ils sont « sans personnalité ». D'où le rayonnement lumineux et exceptionnel d'une apparition comme K. avec «son âme insoumise » qui « ressent avec sollicitude et passion tout ce qui est commis dans ce monde »[3.7.1940].

\section{Le régime face à l'« émigration intérieure » : une nouvelle constellation ?}

Si en 1933-1934 le retrait dans une vie privée secrète semblait encore un phénomène marginal, cette attitude s'est largement répandue dans l'entourage de Man'kov à la veille de la guerre. D'où vient une telle attitude ? Quel est son enjeu ? Lui reste-t-il un quelconque potentiel politique ?

O. Kharkhordin voit dans la dissimulation l'acte constitutif de la coupure entre public et privé en Union soviétique. Il renvoie notamment aux vydvižency, ces paysans devenus ouvriers puis promus à de hautes responsabilités, qui furent massivement confrontés (par les Commissions de contrôle) à des normes de conduite et des exigences morales qu'ils avaient du mal à s'approprier. Alors que le discours officiel prônait l'autoéducation, l'autoentraînement, la culture individuelle, il leur était beaucoup plus facile d'éviter les pratiques dénoncées uniquement dans la sphère publique. À la différence de la dissimulation au sens d'hypocrisie (licemerie, changer de visage), la pratique en question correspondrait plutôt à pritvorstvo et pritvorjati, i.e. « fermer la porte ». Si auparavant il n'y avait pas de sphère privée proprement dite, selon Kharkhordin cette pratique de dissimulation est à la base d'une nouvelle sphère particulière, bien distincte de la sphère privée occidentale (comme espace protégé par la loi), qui, dans la mesure où elle est secrète, est créée indépendamment par les individus. À la différence de la dissimulation collective pratiquée durant des siècles par les paysans vis-à-vis des autorités (représentants du tsar, collecteurs d'impôts, etc.), c'est une pratique individuelle qui est, selon Kharkhordin, à l'origine de la figure de

33. Une autre fois, il note de la même veine : «9.3.1939 Réunion des membres de tous les cercles scientifiques. J'ai fait un discours sur l'expérience du travail de mon cercle. J'ai quelque peu enjolivé. Et, en général, à cet égard je me suis fait une réputation qui est presque ridiculement fausse. » 
l'individu en Union soviétique. Cet individu, différent de son homologue occidental, préfigurerait la « raison cynique » du socialisme soviétique tardif. La dissimulation serait ainsi la seule pratique que les citoyens soviétiques auraient découverte et effectuée de façon complètement indépendante ${ }^{34}$.

Cette genèse vaut peut-être pour partie de la population, mais il nous semble que les signes de connivence que Man'kov observe dans son entourage universitaire suggèrent une autre origine à ce repli sur soi. À en juger par les remarques du journal, il semble qu'au lieu de «fermer leurs portes » les gens ont plutôt tendance à les entrouvrir. C'est, certes, pour jeter un coup d'œil sur les autres, mais aussi pour les laisser regarder dans leur petit univers à part, au-delà (ou en deçà ?) de cette façade publique. N'est-ce pas cet acte de communication non verbale, la voix oblique, qui contribue largement à constituer ces petits univers ? Et n'est-ce pas là une pratique intersubjective plutôt qu' « individuelle », un engagement témoignant d'une recherche de garanties, de petites mises en commun pour matérialiser des doutes vagues et un mécontentement diffus par un ancrage dans une réalité partagée ?

C'est supposer qu'une grande majorité de gens voit dans ce nouveau résidu d'une existence intime et secrète, au-delà de l'engagement politique obligatoire, un gain de liberté personnelle, et qu'ils se contentent d'avoir conquis un espace dont la fragilité - épreuve douloureuse des années passées - les conduit à veiller surtout à ce qu'il ne dépasse pas ses étroites limites et reste inaperçu des organes de contrôle et de ses nombreux agents. Mais d'un autre côté on voit que le régime se rend compte progressivement que ses appuis inconditionnels (les «orthodoxes » limpides) sont en train de s'effondrer, et que la naissance et l'augmentation de tels espaces particuliers ne permet plus d'assurer une surveillance omniprésente, d'autant que les agents jusqu'alors loyaux commencent à créer de tels espaces de refuge pour eux-mêmes. L'exigence officielle d'univocité - manifestée dans la chasse aux « gens à double face » (dvurušniki) - est ainsi minée de l'intérieur et le régime a surtout intérêt à trouver un moyen pour endiguer le potentiel subversif de ces espaces, si évident chez Man’kov, mais aussi dans le conte de L.

Ce n'est pas le repli sur soi qui est redouté par les autorités staliniennes, mais en tout premier lieu le fait que les gens entrouvrent les portes, précisément en raison du potentiel politique de cet échange, surtout si les interlocuteurs mécontents font de plus en plus état de leur désaccord avec la « façade » de la propagande. À en juger par les observations de Man’kov, (sur les festivités du 1 1 ${ }^{\text {er }}$ mai par exemple), ce ne sont plus seulement les individus qui «ferment leurs portes », mais le régime qui se hâte de les fermer de son côté, plutôt que de prendre le risque que les « habitants » les ouvrent entièrement. La kommunalka, jusqu'alors moyen de surveillance bienvenu (bien que né de la pénurie de logements), devient de plus en plus suspecte précisément à cause de cette évolution. D'où aussi les investissements considérables dans la construction de nouveaux logements dans l'après-guerre, logements

34. Voir Oleg Kharkhordin, The Collective and the Individual in Russia: A Study of Practices, Berkeley-Los Angeles-Londres University of California Press, 1999 p. 270-71 qui explique ainsi les changements sociaux au moment de l'effondrement de l'URSS. 
dont les locataires sont désormais censés fermer les portes derrière eux au lieu d'avoir un accès permanent à des pièces partagées, endroits susceptibles de favoriser la mise en commun d'un mécontentement ou d'un désaccord. Pour le régime stalinien, il est préférable qu'ils s'adonnent à une existence recluse de «petitbourgeois », bien distincte de leur existence publique, que de vivre pleinement un potentiel politique qui comporte toujours le risque qu'ils ne se retournent contre la politique du régime en place pour accorder engagement public et pensées politiques secrètes...

La nouvelle logique d'une existence divisée en engagement communiste en public et valeurs «petites-bourgeoises $»^{35}$ dans une vie personnelle détachée s'instaure en silence, mais avec le concours du régime. Celui-ci, devenu de plus en plus craintif, préfère cette logique de l'indifférence dissimulée et du petit gain personnel à l'engagement révolutionnaire «total » et sans compromissions : cet engagement ne risque-t-il pas de se retourner résolument contre le système en place, dès que celui-ci est considéré comme entrave à la révolution ? Voilà un aspect important, bien que jusqu'ici négligé, qui aboutit à ce que Vera Dunham a appelé le big deal entre le régime et ses sujets, ce compromis tacite qu'elle a repéré dans la littérature de l'après-guerre ${ }^{36}$. Ainsi est rendu possible que dans un roman comme Le premier et le dernier (d'ailleurs de peu de valeur sur un plan littéraire) l'ingénieur enthousiaste finit par être dégradé en fin de compte pour sa sincérité. Il refuse de s'engager dans le social en plus - et au détriment - de son travail d'ingénieur, persuadé que c'est ainsi qu'il sert au mieux la communauté. Mais on exige de lui de faire au moins semblant de se plier aux règles du jeu, c'est-à-dire de faire une déclaration creuse, convenue uniquement afin de consolider la façade du discours officiel. Pour le protagoniste (un antihéros !) une telle tricherie ne peut se concilier avec sa conscience, ce qu'il paie ${ }^{37}$.

Le régime semble avoir de plus en plus intérêt à pousser les gens vers l'hypocrisie et, par ce biais, vers une sorte d'«émigration intérieure », non pas qu'il craigne une résistance ouverte immédiate, mais parce qu'il redoute le potentiel critique de la communication qui est la condition de la possibilité même de résistance. En s'attaquant aux liens personnels en tant qu'espaces de communication, le régime cherche donc à étouffer en germe toute résistance potentielle, au lieu de se contenter de faire face à des problèmes de résistance réelle ${ }^{38}$.

35. C'est le terme employé par Man'kov pour critiquer ses contemporains qui font preuve de pusillanimité (la dépolitisation que nous venons d'esquisser) sans même tenter une mise en commun alternative susceptible d'aboutir à une nouvelle politisation et, en fin de compte, à une mise en cause du régime.

36. Vera S. Dunham, In Stalin's Time: Middleclass Values in Soviet Fiction, Cambridge-New York : CambridgeUniversity Press, 1976.

37. Voir p. ex., le roman de S. Marvič, Pervyj i poslednyj [Le premier et le dernier], Zvezda, 4 (1949), p. 87, cit. d'après Dunham, op. cit., p. 203.

38. Cet aspect est abordé plus en détails dans notre thèse : Malte Griesse, Communiquer, juger et agir sous Staline. La personne prise entre ses liens avec les proches et son rapport au système politico-idéologique, Thèse dedoctorat, P., EHESS, 2008. 
C'est là une nouvelle forme d'atomisation qui n'est plus effectuée brutalement par la Terreur, mais implicitement imposée par le régime. Contrairement à ce que dit Man'kov de façon très générale sur la décomposition de la société qui ne se limiterait pas à la famille, il semble que ce big deal vise moins un repli sur un soi complètement atomisé qu'à un repli sur la famille nucléaire ${ }^{39}$, unité que les besoins fondamentaux de la survie rendent bien moins susceptible (que les amitiés, p. ex.) de générer des «montées en généralité » (critiques) et des engagements publics. ${ }^{40}$ Après la politique contradictoire des années 1930 vis-à-vis des liens familiaux, le régime cherche ainsi de plus en plus à renforcer la famille dans ses fonctions de base ; ses préoccupations primaires sont considérées comme relativement inoffensives du point de vue de son potentiel à engendrer de la communication sur des sujets politiques et d'invoquer ces objectifs révolutionnaires communs qui pourraient rivaliser avec le monopole que le régime s'est arrogé quant à l'héritage légitime de la révolution et de ses valeurs universelles. Ce jugement de la famille comme espace de communication plutôt apolitique se confirme dans le cas de Man'kov : les deux frères sont proches dans leurs propos critiques mais cela ne débouche jamais sur des échanges à ce sujet sur une mise en commun entre eux.

Si le régime pousse de plus en plus, même ses partisans orthodoxes, à trouver une vie individuelle ou familiale au-delà de l'engagement politique, Man'kov comme d'autres critiques de sa trempe cherchent au contraire à les arracher à cet espace de réclusion, les encourager à se réunir, à parler horizontalement afin d'agir verticalement contre l'hypocrisie, la façade et le mensonge. Ils partent de liens amicaux, rarement d'espaces familiaux.

\section{Université de Bielefeld}

Centre Marc Bloch, Berlin

malte.griesse@uni-bielefeld.de

39. Voir les observations de Sheila Fitzpatrick, Tear off the Masks! Identity and Imposture in Twentieth-CenturyRussia, Princeton, NJ : Princeton Universit Press, 2005, p. 240-261 sur la politique de la famille dans l'après-guerre.

40. Le concept de la «montée en généralité », d'une justification ou critique qui fait appel à un bien public (oupotentiellement public) s'appuie sur la sociologie pragmatique de Boltanski et Thévenot. Voir Boltanski,Thévenot, De la justification... 